(Birdem Med J 2013; 3(1):66)

\title{
Academic Achievement
}

\begin{tabular}{|clll|}
\hline Sl. No. & Name & Academic position & Academic Achievement \\
\hline 1. & Dr. Farhana Afroz & SMO (Internal Medicine) & FCPS (Medicine) \\
\hline 2. & Dr. Erfanur Rahman Shuvo & Registrar (Haemodialysis) & FCPS (Medicine) \\
\hline 3. & Dr. Sarker Mohammad Sajjad & SMO (GHPD) & MRCP(UK) \\
\hline 4. & Dr. Shakila Khanum & MO (Obstetrics \& Gynaecology) & MCPS (Obstetrics \& Gynaecology) \\
\hline 5. & Dr. Shala Mahazabin & SMO (Surgery) & MS (General Surgery) \\
\hline
\end{tabular}

